\title{
Design of Cyber-Physical Systems Architecture for Prognostics and Health Management of High-speed Railway Transportation Systems
}

\author{
Zongchang Liu ${ }^{1,2}$, Zhiqiang Zhang ${ }^{3}$, Guanji Xu ${ }^{3}$, Wenjing Jin ${ }^{2}$, Jay Lee ${ }^{1}$ \\ 1, NSF Industry/University Cooperative Research Center on Intelligent Maintenance Systems (IMS), University of Cincinnati, \\ Cincinnati, OH, United States \\ ${ }^{2}$ CyberInsight Technologies Co. Ltd., Beijing, China \\ ${ }^{3}$ CRRC Qingdao Sifang Co., Ltd., Qingdao, China \\ liuzc@mail.uc.edu \\ zhangzhiqiang@cqsf.com
}

\begin{abstract}
The high-speed railway (HSR) transportation system in China has been growing rapidly during the past decade. In 2016, the total length of HSR in China has reached to 22,000 kilometers, and there are over 2,000 pairs of high speed trains operating daily. With the advancement of design and manufacturing technologies, the reliability and construction costs have been improved significantly. However, there is still great need for reduction of their operation and maintenance costs. With such incentive, a pilot project has been launched to develop a prognostics and health management system for rolling stock to transform the maintenance paradigm from preventive to predictive maintenance. Considering the high task variety and big data environment in HSR real-time monitoring system, a cyberphysical system (CPS) architecture is proposed as the framework for its PHM system. This paper reviews the needs of predictive maintenance for the HSR system, and then present a concept design of the CPS-enabled smart operation and maintenance system.
\end{abstract}

\section{INTRODUCTION}

The high-speed rail (HSR) system in China has been growing rapidly since 2007. In 2016, there were over 2,000 pairs of high speed trains operating daily with total ridership of 1.4 billion, making the HSR China most heavily used in the world. A recent survey conducted by Nomad Digital among rail operators shows that operation and maintenance costs

\footnotetext{
Zongchang Liu et al. This is an open-access article distributed under the terms of the Creative Commons Attribution 3.0 United States License, which permits unrestricted use, distribution, and reproduction in any medium, provided the original author and source are credited.
}

have become the biggest concern (Feigenbaum, 2013), and China is no exception. For example, the maintenance paradigms of HSR in China is mainly preventive maintenance with large safety margin due to the high safety standard, which has caused considerable cost burden and challenges of sustainable growth of China's HSR system; Meanwhile, many Europe countries such as Italy, France all have high safety requirements on their railway transportation systems and also encourage to reduce maintenance costs. Thus, it has attracted many attentions on new techniques for railway condition-based maintenance. These concerns have led to the development of Prognostics and Health Management (PHM) solutions in CRRC Group to transfer the condition monitoring data into desired information and knowledge to improve the life-cycle costs and service reliability (Lee, 2015).

The enabling technologies to develop a PHM system involve condition monitoring, information and communications technologies (ICT), and more importantly predictive analytics technologies. The primary goal is to transform the invisible patterns of component degradation and loss of efficiency into health insights (Lee, 2015). A good example of PHM system practice in HSR is the TrainTracer ${ }^{\mathrm{TM}}$ launched by ALSTOM in 2006 for real-time remote monitoring of trains as reported by Worth et al. (2014). Lu et al. (2016) introduced another product named TrackTracer ${ }^{\mathrm{TM}}$ has also been developed by ALSTOM that is complementary to and integrated with TrainTracer ${ }^{\mathrm{TM}}$ to further enable predictive maintenance service for track infrastructures. 
It has been has realized that manufacturing of rolling stocks solely has a predictable limit of market volume and profit margin. Therefore, predictive analytics and smart services have been introduced for enhancing the product competency and improving the sustainability of value chain. This paper discusses a framework design of predictive maintenance system based on the architecture of Cyber-Physical systems.

\section{PHM FRAMEWORK DESIGNED BASED ON CYBER-PHYSICAL SYSTEMS}

The concept of cyber-physical systems (CPS) has been defined as an engineered system in which the nature and human made systems (physical space) are integrated with computation, communication, and control systems (cyber space) in all scales (Incorporated, 2013). Lee, et al. (2013) proposed an implementation framework of CPS for predictive maintenance which consists of five levels. Inspired by the five-level architecture design of CPS, this paper proposes a framework for rolling stock predictive maintenance, which consists of the following elements, as seen in Figure 1.

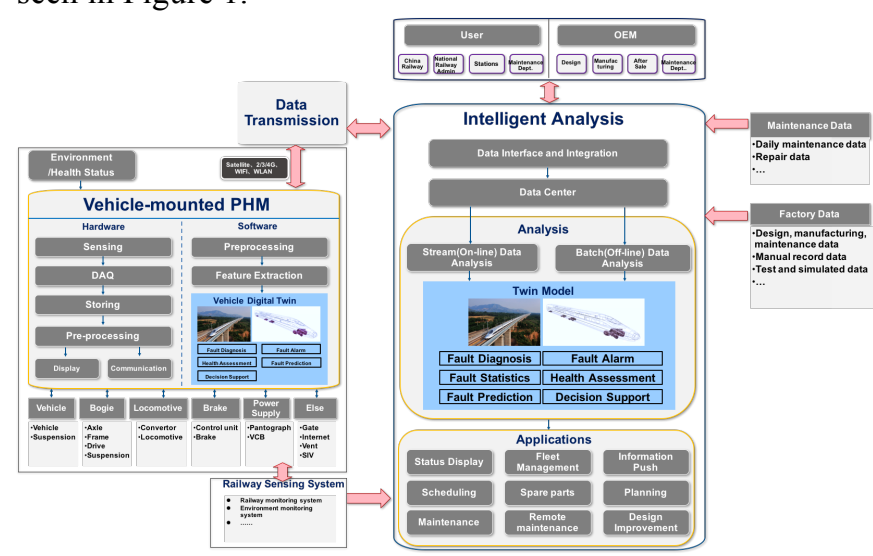

Figure 1: The PHM System Framework Design

Data connection: There are various data sources from the rolling stock and the operating environment. Data sources related to operation conditions includes add-on sensors and controllers from critical subsystems, which include traction motor, power transmission systems, bogies and electronic systems. Railway infrastructure such as tracks, catenaries and point machines can also be sources of data that needs to be integrated in the onboard DAQ system.

From data to health features: After connecting various data sources in the train, the data will be further converted to health related features in the onboard processing system. Signal processing, feature extraction, health assessment \& diagnosis algorithms (such as self-organizing map, logistics regression, support vector machines, etc.) and predictive analytics are integrated in a train-based analytical server; The health information is then used to support the decision of drivers to be aware of the condition and potential risks.

Data mining and modeling: The underlying features and selected raw data from fleets of trains are transferred to a data center. With a big data environment created, data mining for knowledge discovery and model development will be performed with advanced algorithms. Peer-to-peer comparison, information sharing, collaborative modeling, and time machine records of utilization matrix and health condition history will also be developed. Models created or improved in the data center is able to be deployed and updated to multiple fleets.

Decision support Apps: In this level, the PHM analytics results of the railway system will be combined with the expert knowledge through their inputs for making optimal fleet management and maintenance decisions. The analytics results and decision support information will be shared in Web service APPs to different sectors, such as the HSR operators, OEMs, and service providers.

\section{A PREDICTIVE ANALYTICS INTERFACE TO CONNECT PHYSICAL AND CYBER SPACE}

For HSR systems, one could consider a variety of critical assets in the physical space, such as locomotive induction motor, bogies, wheelset bearings, and transmission gearbox. With various components and data sources, it urges to consider what hidden issues of the assets are of most concern, and what invisible information needs to be predicted and revealed in cyber space. For instance, the development of winding shortage is difficult to be measured directly, but could be monitored from sequence impedance features which are extracted from motor current and voltage signals. Therefore, appropriate features and analytical models need to be established in a cyber-physical interface to enable transparency of the hidden state in physical space as twin model representations in cyberspace, as indicated in Figure 2. The CPI introduces the concept of 'Time Machine' to convert the continuous and heterogeneous data source into structured data format for further computation. To improve data storage and computation efficiency, the snapshots of sensor data, controller data, and event data are all recorded instead of the raw time-series data. These snapshots are only taken in discrete time, and can be triggered by either fixed time intervals, or based on event such as change of operation regimes and health status. During the lifecycle of a machine, these snapshots will be accumulated to construct the 'time machine' history of condition changes of the assets. Each 
snapshot record can be used as a twin model of a particular condition for peer-to-peer comparison and causal relationship modeling between the assets.

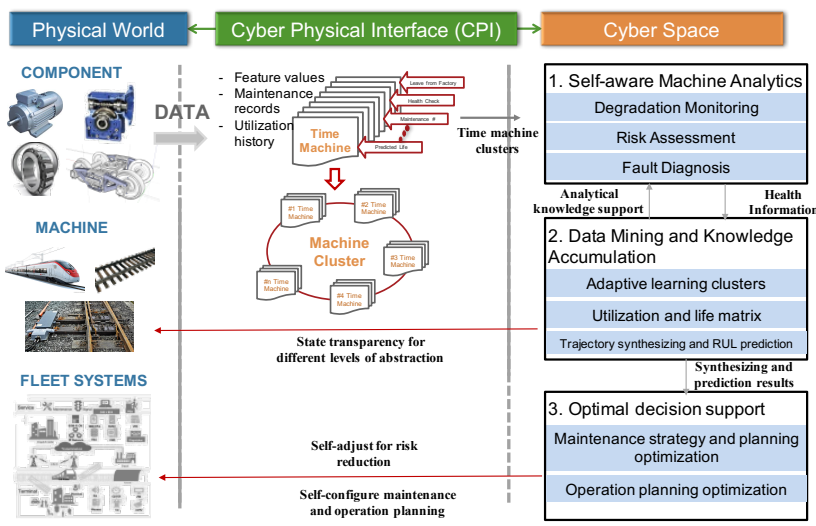

Figure 2: Cyber-Physical Interface Platform for HRS Applications

\subsection{Introduction to Time Machine}

The Cyber-Physical Interface (CPI) first introduce the concept of 'Time Machine' to convert the continuous and heterogeneous data source into structured data format for further computation. To improve data storage and computation efficiency, snapshots of sensor data, controller data, and event data are recorded instead of the continuous time-series data. This step usually involves signal processing and feature extraction techniques in different domains. These snapshots are only taken in discrete time, and the can be triggered by either fixed time intervals, or based on event such as change of operation regimes and health status. During the life-cycle of a machine, these snapshots will be accumulated to construct the 'time machine' history of operation and condition changes of the particular assets. Each snapshot record can be used as a particular machine at a particular condition for peer-to-peer comparison and causal relationship modeling between the assets. An illustration of Time Machine approach for cyber-physical interface is presented in Figure 3.

\subsection{Adaptive Clustering for Self-aware Machine Analytics}

A challenge for effective and efficient snapshot collection to construct the 'time machine' of monitored asset is to determine the state of each snapshot. The state could be defined as possible working regimes, machine condition, degradation pattern, and failure modes. In addition to classify the snapshots with known state, it should also identify new state that has not been observed to enable self-awareness and self-learning capabilities of CPS.

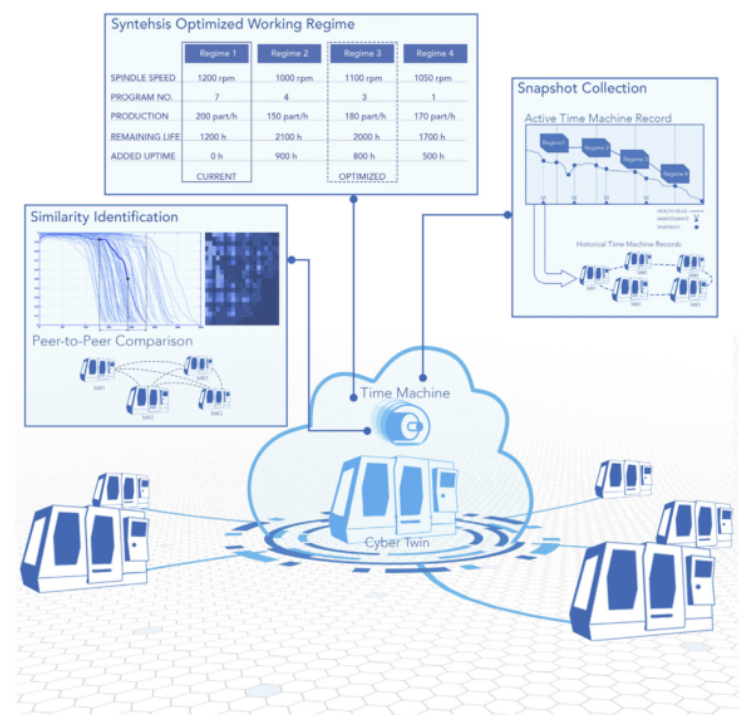

Figure 3: Time machine approach for CPI

For fleet assets that perform similar tasks under similar health conditions, the symptoms in the feature space should also be similar. Under this assumption, the snapshots of fleet assets over time should have clustering tendencies, where snapshots at similar state tend to gather together in feature space. Unsupervised learning algorithms such as self-organizing map, discriminant diffusion maps, and Laplacian Eigenmaps can be used for autonomously creating clusters for different working regimes and health conditions. The framework illustrated in Figure 4 provides an online updating mechanism for state awareness of monitored asset (Lee, 2015). The algorithm compares the latest snapshot with the existing clusters, and afterwards, the state is updated in two scenarios: (1) the snapshot is assigned to an existing cluster, and the is labeled as having the same state of the identified cluster; and (2) there is no similar cluster found, and the algorithm will hold this sample until it sees enough counts to generate a new cluster.

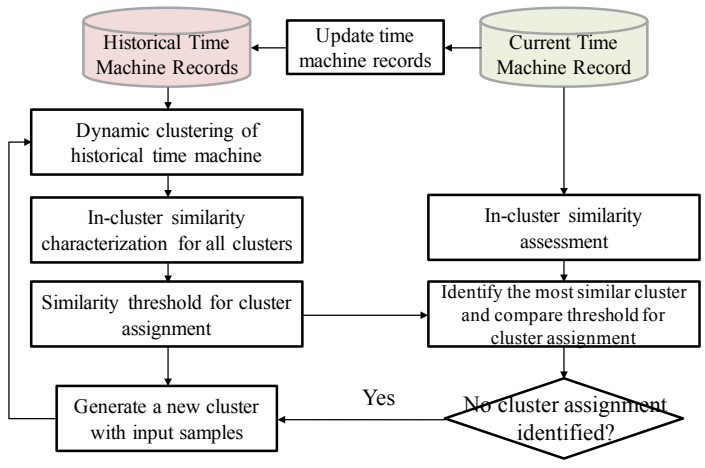

Figure 4: Adaptive clustering for self-awareness of machine state 


\subsection{Fleet-based Analysis for Enhanced Prognosis}

Unlimited existence of twin models results in continuous accumulation of time machine records and consequently gathering clusters of identical components in time horizon. The time machine records in cyberspace are discrete and structured, which allows comparison and clustering from different dimensions. There are generally three dimensions of comparison between the time machine records. To compare the same unit in time horizon is the most widely adopted method for fault prognosis. In such process, the degradation of the unit is monitored by performing an objective comparison between the latest input vector and baseline model, and early warning of fault can be triggered when the variation has exceeded a given threshold. Beyond that, the comparison can also be performed between units at similar operation regimes. Based on similarities between machine conditions, clustering methods can be applied to group the machines under similar conditions to generate local baselines. Lapira (2012) investigated the use of clustering algorithms and peer-to-peer comparison approach for fault detection in a fleet of assets, such as wind turbines and industrial robots. Moreover, peer-to-peer comparison can provide performance ranking information to prioritize maintenance actions and balance load stress between a network of machines. Last but not least, the time machine trajectory can also be compared between units to improve the accuracy of RUL prediction and performance synthesizing. The goal is to accumulate a library of time machine trajectories, and when the time machine records over time of the current asset is obtained, prediction of future performance is done by measuring the pairwise similarity between the current trajectory and a snapshot of the trajectories in the library. The prediction of future trend for the current trajectory is a weighted ensemble of the best matching trajectories in the library. Successful implementation of this concept includes RUL prediction of jet engines (Wang, 2008) and performance prediction in manufacturing process (Liu, 2007).

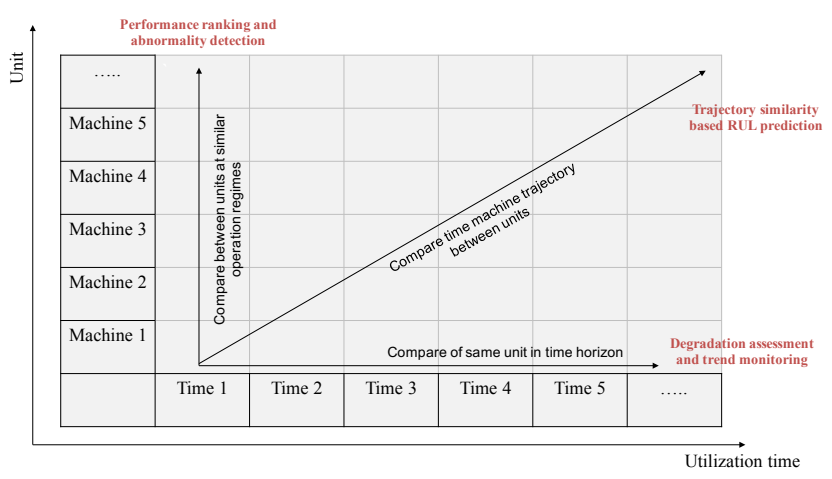

Figure 5: Peer-to-peer comparison horizons and their purposes

\section{CASE STUDY}

Induction motor is one of the major component of the rolling stock. Winding insulation degradation is a critical failure mode in the health management of locomotive induction motors. In this case study, current and voltage signals from the three phases of the induction motors were collected from an in-service high-speed train. As the signature of the machines may differ significantly during different operational regimes, it is necessary to divide the data samples into clusters according to the working regime under which each sample data was collected. A frequency-domain based technique was used to estimate the motor speed during data collection. This was done by tracking the location of the main peak in the motor current spectrum, in which the frequency is related to the speed of the train.

Negative-sequence impedance is an effective parameter for evaluating the symmetry of the current and voltage in a three phase system. It is calculated by computing the symmetrical components of both current and voltage in a three-phase system. In this case study, the negative-sequence impedance feature was calculated when the motors were in constant speed, and was used to derive the indicator to infer the health condition of motor winding. In terms of fault detection, peerto-peer comparison of the negative impedance features between the motor fleet is proved to have better detection accuracy. Figure 6 shows the results of the analysis for motors of two locomotives, and the motor with winding fault damage is labeled in red. 

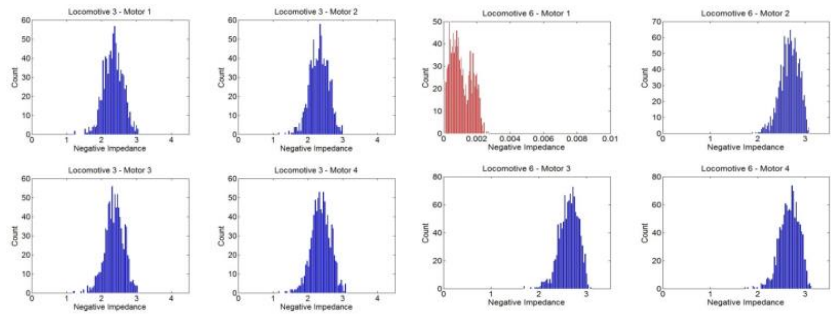

Figure 6: Distribution of negative impedance of healthy and abnormal locative motors

\section{Conclusion}

In this paper, the experience of HSR condition monitoring and concepts of CPS are combined to consider a framework of PHM system architecture and discuss the elements in the framework. The PHM system architecture proposed in this paper is designed to meet the requirements and challenges in large fleets and big data environment. A train-based agent with smart analytics algorithms is used to collect data and convert them to health related information. The underlying features and selected raw data are further transferred to the data center for knowledge discovery, model development, and decision support. The health insights and decision references can be further used by different sectors of the HSR industry through App services to improve collaborative optimization and synchronization.

\section{ACKNOWLEDGEMENT}

This research is funded by the CRRC key project of Railway System Prognostics and Health Management. The authors would like to express gratefulness to CRRC Qingdao Sifang Co. Ltd. for their sponsorship and technical support.

\section{REFERENCES}

N. Digital (2015). Rail industry survey: Major challenges facing rail operators, maintainers \& owners and the role of ICT. Rail industry survey.

B. Feigenbaum (2013). High-Speed Rail in Europe and Asia: Lessons for the United States.

J. Lee, H. D. Ardakani, S. Yang, and B. Bagheri (2015). Industrial big data analytics and cyber-physical systems for future maintenance \& service innovation. Procedia CIRP, vol. 38, pp. 3-7.

J. Lee, H. Kao, and S. Yang (2014). Service innovation and smart analytics for Industry 4. 0 and big data environment. Procedia CIRP, vol. 16, pp. 3-8.

A. T. S. Worth and B. Escartin-Claveria (2014). Alstom's approach to railway condition monitoring. 6th IET Conference on Railway Condition Monitoring Birmingham, 2014. pp. 1-6. doi: 10.1049/cp.2014.0991.

Lu X., Shan S., Tang G., Wen Z. (2016) Survey on the Railway Telematic System for Rolling Stocks. In: Qin
Y., Jia L., Feng J., An M., Diao L. Proceedings of the 2015 International Conference on Electrical and Information Technologies for Rail Transportation. Lecture Notes in Electrical Engineering, vol 378. Springer, Berlin, Heidelberg

E. Incorporated (2013). Foundations for Innovation in CyberPhysical Systems.

H. Kao, W. Jin, D. Siegel, and J. Lee (2015). A Cyber Physical Interface for Automation SystemsMethodology and Examples. Machines, vol. 3, pp. 93106.

J. L. Behrad Bagheri, Shanhu Yang, Hung-An Kao (2015). Cyber-physical Systems Architecture for Self- Aware Machines in Industry 4.0 Environment Cyber-Physical Environment. International Federation of Automatic Control, 2015, no. January, pp. 1622-1627.

E. R. Lapira (2012). Fault Detection in a Network of Similar Machines using Clustering Approach.

T. Wang, J. Yu, D. Siegel, and J. Lee (2008). A SimilarityBased Prognostics Approach for Remaining Useful Life Estimation of Engineered Systems. 2008 International Conference on Prognostics and Health Management, pp. 4-9.

J. Liu, D. Djurdjanovic, J. Ni, N. Casoetto, and J. Lee (2007). Similarity based method for manufacturing process performance prediction and diagnosis. Computers in Industry, vol. 58, pp. 558-566. 